\title{
A legacy role for DNA binding of Lon protects against genotoxic stress
}

\author{
Rilee D. Zeinert ${ }^{1}$, Jing Liu ${ }^{1}$, Qiyuan Yang ${ }^{2}$, Yunguang Du ${ }^{2}$, Cole M. Haynes ${ }^{2}$, Peter \\ Chien $^{1 *}$
}

\section{Affiliations:}

${ }^{1}$ Department of Biochemistry and Molecular Biology, Molecular and Cellular Biology Program, University of Massachusetts Amherst, Amherst MA

${ }^{2}$ Department of Molecular, Cell and Cancer Biology. University of Massachusetts Medical School, Worcester MA

*pchien@biochem.umass.edu 


\begin{abstract}
:
The quality control protease Lon was originally characterized as a DNA binding protein, yet the physiological consequences of this interaction are not understood. Here we use the $\alpha$-proteobacteria Caulobacter crescentus to show that DNA binding of Lon is critical for DNA damage tolerance. In vitro, DNA can directly activate or inhibit Lon activity and promotes degradation of DNA bound proteins by neighboring bound Lon. Bacteria expressing a DNA-binding deficient Lon variant are phenotypically wildtype with respect to normal growth and response to proteotoxic stresses, but are sensitive to genotoxic stresses. Disrupting Lon binding to mitochondria genomes also results in sensitivity to DNA damage but otherwise maintained normal mitochondrial function, consistent with the bacterial ancestry of this organelle. We propose that clearance of overly persistent proteins from DNA, including DNA-protein crosslinks, by the Lon protease is an important damage response that originated in free-living $\alpha$-proteobacteria and has been preserved during the endosymbiotic transition to mitochondria.
\end{abstract}

One Sentence Summary: DNA binding by the Lon protease protects against genotoxic damage in a manner that has been preserved from bacteria to mitochondria. 


\section{Main Text:}

Caulobacter crescentus (Caulobacter) is a freshwater dimorphic a-proteobacteria that undergoes a developmental transition during its cell cycle (1). Energy dependent proteolysis by $\mathrm{AAA}+$ proteases drives this cell cycle (2). For example, the ordered degradation of cell cycle regulators by the CIpXP protease requires the hierarchical assembly of adaptors (3-9). In Caulobacter, the Lon protease degrades the epigenetic regulator $\operatorname{CcrM}(10)$, the transcriptional regulator $\operatorname{SciP}(11)$, and the replication initiator DnaA(12). In E. coli, Lon is responsible for degrading misfolded proteins as part of quality control $(13,14)$, but one of the original phenotypes of lon was sensitivity to DNA damage, exemplified by Evelyn Witkin's original studies using the naturally lon deficient B strain to define the genetic basis of radiation resistance (15). Subsequent suppressor studies revealed that degradation of the E. coli cell division inhibitor SulA by Lon is responsible for a large part of this sensitivity $(16,17)$. Whether Lon plays a role in DNA damage in other bacteria, such as Caulobacter, remains poorly understood. In mitochondria, Lon has been clearly implicated in managing the response to protein misfolding stresses although it is unclear how it might affect mtDNA stress (18-20). We sought to address these questions beginning from a biochemical perspective.

Although Lon has long been known to bind DNA $(21,22)$, there are conflicting reports on whether DNA acts in an inhibitory or activating role (23-25). Using a FITC-casein degradation assay and purified Caulobacter Lon protease, we found that DNA clearly modulates Lon activity, but that the outcome of DNA binding is not easily predicted. Genomic DNA isolated from Caulobacter inhibits Lon activity, while short dsDNA oligonucleotides or PCR products elicited a more minor effect (Figure 1). By far, the most dramatic variation was seen with single stranded DNA oligonucleotides: some activate Lon $\sim 2$-fold and others repress activity $>5$-fold (Figure 1). Because casein is not 
known to bind DNA, our data suggests that DNA can both directly activate or inhibit general Lon protease activity. Analyses of these oligonucleotides did not show any strong correlation between activation/inhibition and specific sequences, length, dinucleotide motifs, or $\mathrm{T}_{\mathrm{m}}$ (Figure $\mathrm{S} 1$ ). However, there was a strong correlation in the strength of either inhibition or activation with the predicted ability to form G-quadruplex (G4) structures (Figure 1,S1).

G4 structures are noncanonical DNA structures with unknown biological functions, but are associated with changes in transcription, replication and DNA damage (26). Interestingly, G4 favoring sequences have been previously associated with binding to human mitochondrial Lon $(20,27)$, but the consequence of this interaction on Lon activity was not determined. To validate this correlation with G4 structures, we focused on a strongly inhibitory oligonucleotide (OPC498) which contains a predicted hairpin structure and G4-favoring region. Mutating the hairpin structure did not affect the inhibitory activity (Figure 1) nor binding to Lon (Figure S1), but eliminating the G4 region resulted in loss of inhibition and Lon binding (Figure 1, S1). A G4-containing sequence known to bind the human mitochondrial Lon (20) also inhibits protease activity (Figure 1). Our conclusion is that while Lon can bind many DNA sequences, it has a particularly affinity for guanine rich sequences that can form G4 structures.

One proposed role for DNA binding of Lon is that it could promote degradation of DNA bound proteins (28-30). We tested this hypothesis by using DnaA, a known in vivo substrate of Lon that is poorly degraded in vitro in the absence of Lon regulators (12). In Caulobacter, DnaA binds to origin DNA most strongly through G-box motifs (31). We used a dsDNA containing the first origin G-box (G1box) and the natural downstream guanine-rich region that we predicted would bind Lon. As expected, Lon alone degrades 
DnaA poorly, but addition of the G1box dsDNA dramatically accelerates degradation

(Figure 1). Both the features are important as mutation of the G-box or removal of the guanine-rich region eliminate the enhanced degradation (Figure 1). We hypothesized the G1box dsDNA is acting as a simple scaffold and tethers DnaA with Lon to drive proteolysis. If so, then excess scaffold should counterintuitively suppress DnaA degradation because the ternary Lon-G1box-DnaA complex will be depleted when pairwise Lon-G1box and DnaA-G1box species accumulate at high scaffold concentrations. Consistent with our model, addition of excess dsDNA initially activates substrate degradation ( $\mathrm{K}_{\text {activation }} \sim 300 \mathrm{nM}$; Supp Info), but inhibits at higher concentration (Figure 1).

In order to explore the specific role of DNA binding in Lon regulation, we required a Lon variant that was incapable of binding DNA, but otherwise active. Based on previous work in E. coli Lon (32), we mutated a cluster of lysines (K302, K304, K306, K307) to glutamic acids to generate a DNA binding deficient Lon, Lon4E. Purified Lon4E degrades FITC-casein with similar kinetics as wildtype Lon, but is insensitive to inhibition by OPC498 (Figure 2). $\Delta$ /on Caulobacter exhibit profound morphological defects including cell body elongation, increased stalk organelle length and loss of motility in soft agar (10)(Figure 2). Importantly, cells expressing only Lon4E rescue these phenotypes (Figure 2; Figure S2), confirming our in vitro results that Lon4E is fully active. Loss of Lon results in increased levels of its substrates DnaA and $\operatorname{CcrM}(10,12)$ and Lon4E largely restores steady-state levels to wildtype (Figure 2). Degradation of CcrM is also restored by the Lon4E allele (Figure S2). Finally, RNA-seq experiments show that the Lon4E allele does not dramatically alter the transcriptome while loss of Lon has a significant impact on expression of over a hundred genes (Figure S2). We conclude that 
Lon4E complements the function of wildtype Lon during normal, unstressed growth conditions.

We found that in addition to morphological defects, cells lacking Lon were highly sensitive to many stresses (Figure 2). For example, $\Delta$ lon strains grew poorly on media containing L-canavanine, which induces protein misfolding, and when stressed by a variety of DNA damaging agents (Figure 2). Interestingly, Lon4E strains were as resistant to proteotoxic stress as wildtype based on their response to L-canavanine, but were more sensitive to genotoxic stresses (Figure 2). This difference was most dramatic for those agents that induce crosslinking of DNA such as mitomycin C (MMC) or cisplatin (CIS) which must be repaired with complex multistep pathways such as interstrand crosslink repair and translesion synthesis (33). However, minor differences were also present for compounds such as methyl methanosulfonate (MMS), which results in damage that can be resolved by simpler pathways such as base excision or mismatch repair (33). Clearly, loss of Lon results in the most dramatic phenotypes in all stress conditions (Figure 2), suggesting that DNA binding of Lon has a specific role in responding to DNA damaging conditions, but non DNA-dependent Lon protease activity is crucial for stress tolerance in general.

Mitochondria are theorized to have an endosymbiotic origin (34). Specifically, the current model is that a free-living $\alpha$-proteobacterium was engulfed by the ancestor of the modern eukaryotic cell (35). Because Caulobacter is an $\alpha$-proteobacteria, we explored if the need for DNA binding of Lon in tolerating DNA damage was preserved in mitochondria. In C. elegans, mitochondrial Lon (LONP-1) is required for development and growth (19). Based on homology, we engineered a variant of lonp-1 with mutations 
in the corresponding predicted DNA binding residues to generate LONP-1 $1^{4 E}$ and used CRISPR/Cas9 to produce worms expressing only this allele at the endogenous locus. Importantly, we found that worms expressing only the LONP $-1^{4 \mathrm{E}}$ variant at endogenous levels were healthy, grew and developed normally (Figure 3). Because LONP-1 has a known role in mitochondrial protein quality control by recognizing and degrading diverse proteins that incur oxidative damage (18), we infer that LONP- $1^{4 \mathrm{E}}$ is capable of maintaining proteostasis during animal development. Pull-down experiments indicated that LONP-1 robustly binds mitochondrial genomes in vivo as previously suggested(25, 29), but LONP-1 ${ }^{4 E}$ does not (Figure 3). Therefore, DNA binding of mitochondria Lon is not essential for normal development or growth.

The mitochondrial unfolded protein response $\left(U P R^{m t}\right)$ couples the state of mitochondrial protein homeostasis to an upregulation of nuclear encoded chaperone genes. Induction of the UPR ${ }^{\mathrm{mt}}$ requires the transcription factor ATFS-1, which is targeted to the nucleus when mitochondria undergo stress. Complete depletion of LONP-1 activates the UPR ${ }^{\mathrm{mt}}$, as determined by upregulation of the transcriptional mitochondrial chaperone reporter $h s p-6_{p r}: g f p$ (36) (Figure 3) consistent with a role for LONP-1 in the maintenance of mitochondrial function (19). LONP-1 ${ }^{4 \mathrm{E}}$ worms do not activate the UPR ${ }^{\mathrm{mt}}$ suggesting mitochondrial protein homeostasis is intact (Figure 3). However, LONP-1 ${ }^{4 \mathrm{E}}$ worms exposed to cisplatin showed a dramatic activation of UPR ${ }^{\text {mt }}$ that is ATFS-1 dependent while wildtype worms show no response (Figure 3). Importantly, mitochondrial dysfunction driven by depletion of the protein quality control protease SPG-7 caused $\mathrm{UPR}^{\mathrm{mt}}$ activation in both wildtype and LONP-1 ${ }^{4 \mathrm{E}}$ animals to the same degree (Figure 3), as does exposure to paraquat which induces mitochondrial oxidative stress (Figure 3). These results suggest that similar to what is seen in Caulobacter, loss of DNA binding of Lon in mitochondria results in a specific intolerance to genotoxic stress. 
In other work, we had used transposon sequencing in Caulobacter to explore Londependent regulatory pathways and identified genes wherein insertions are synthetically lethal with loss of Lon. One of these genes is uvrD (Figure 4), a helicase originally known for its role in DNA damage repair (37). UvrD has also been shown to be a machine that proficiently evicts proteins bound to DNA, such as disassembling RecA nucleofilaments $(38,39)$ and stalled RNAP complexes $(40)$. Based on our biochemical observation that Lon can degrade substrates via a DNA tether (Figure 1) we speculated that Lon could be particularly useful at degrading overly persistent DNA-bound protein complexes. Therefore, loss of Lon would result in accumulation of proteins on DNA that would be lethal for the cell in the absence of other clearance factors such as UvrD. Consistent with this model, we were able delete $u v r D$ from wildtype cells, but not from $\Delta / o n$ cells (Figure S4) and depletion of Lon in a $\Delta u v r D$ strain results in lethality (Figure 4). We could easily delete other DNA damage response genes ( $\operatorname{rec} A, m m c A, i m u B)$ in a $\Delta / o n$ background to generate viable strains that show no epistasis with respect to genotoxic stresses, suggesting that the synthetic lethality between lon and uvrD is likely due to loss of protein eviction rather than a general consequence of DNA damage intolerance (Figure 4).

Finally, we directly tested if Lon was responsible for clearing persistent protein complexes by evaluating the most extreme persistent case of DNA-protein crosslinks. We used 5-azacytidine, a chemotherapeutic that when incorporated into DNA forms adducts with cytosine methyltransferases resulting in toxic DNA-protein crosslinks (41). Lon was critical for survival in the presence of 5-azacytidine and this toxicity was due to DNA-protein crosslink formation as deletion of the cytosine methyltransferase 
CCNA_03741(42) protects cells lacking Lon from 5-azacytidine (Figure 4). Together, these results support a model where Lon and protein eviction factors (such as UvrD) work together to clear persistent protein complexes, a pathway that is particularly important during complex DNA damage repair.

The role of DNA binding in Lon has been enigmatic since its initial observation $(21,22)$. Previous reports differ on whether DNA binding enhances (24), inhibits (23), or does nothing (25) to Lon protease activity. Our work shows that all outcomes are possible and consistent with earlier work (20) we find that Lon is capable of binding many different sequences, but has preference for sequences with propensity to form G4 structures. Given that G4 structures are associated with DNA damage (26), this selectivity may aid in recruiting Lon to sites where clearance of proteins is particularly important. Our working model is that Lon binding to DNA allows it to recognize neighboring proteins and serves as an additional layer of quality control to reduce the residence time of persistent complexes (Figure 4). This oversight works in concert with protein displacing factors, such as UvrD, and is particularly crucial for irreversibly persistent complexes such as DNA-protein crosslinks (Figure 4). It is noteworthy that repair of DNA-protein crosslinks in eukaryote nuclear genomes have recently been shown to be dependent on DNA binding proteases (43), but no such proteases have been identified in prokaryotes. Our work suggests that Lon can perform this function in bacteria. Finally, we have shown that the same properties of DNA binding in Lon that are important for genotoxic stress responses in Caulobacter are also important in mitochondria, consistent with the endosymbiotic $\alpha$-proteobacteria ancestry of this organelle $(34,35)$. Thus, the legacy of DNA binding in Lon and subsequent protection from genotoxic stress has been preserved through the evolution of the eukaryotes. 


\section{Figure 1}

A.

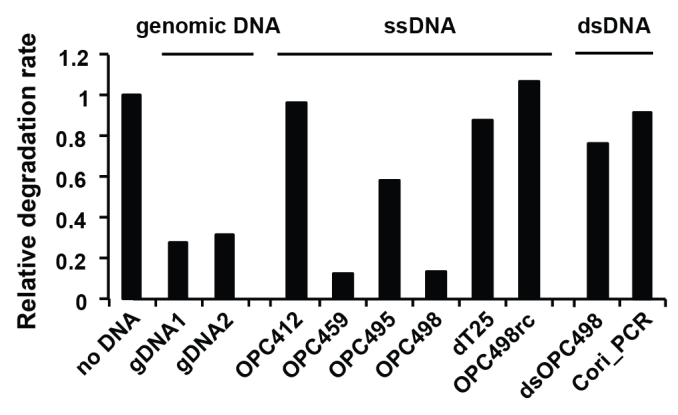

B.

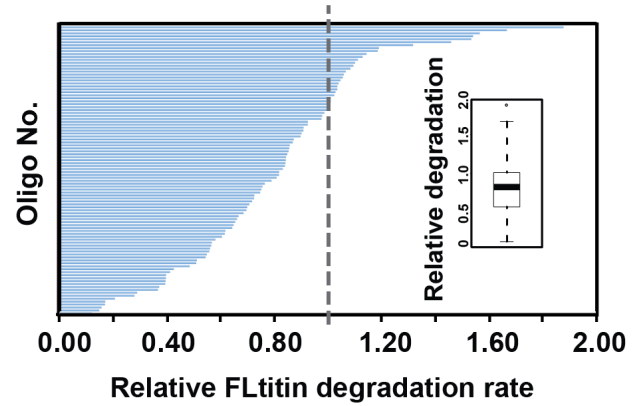

c.

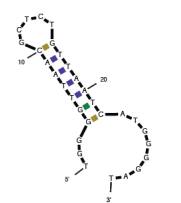

OPC 498



D DnaA

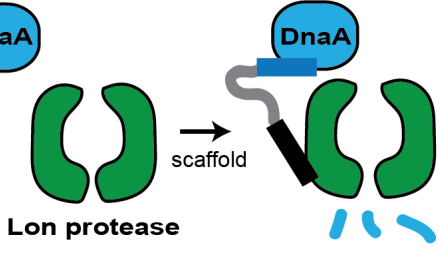

G1 box

DnaA binding

G-rich sequence

AACGGATGATCCACAGGAGAGTCTGGCGCAGGGCGAGAG G1 short

AACGGATGATCCACAGGAGA

G1 null

AACGGAGCGACCCGTGGAGAGTCTGGCGCAGGGCGAGAG

E

no DNA G1 box G1null G1short \begin{tabular}{l|llll|llllllllllll} 
time(min) & 0 & 5 & 10 & 20 & 0 & 5 & 10 & 20 & 0 & 5 & 10 & 20 & 0 & 5 & 10 & 20
\end{tabular}

$$
\begin{gathered}
\text { Lon - } \\
\text { DnaA - } \\
\text { creatine } \\
\text { kinase }
\end{gathered}
$$

F

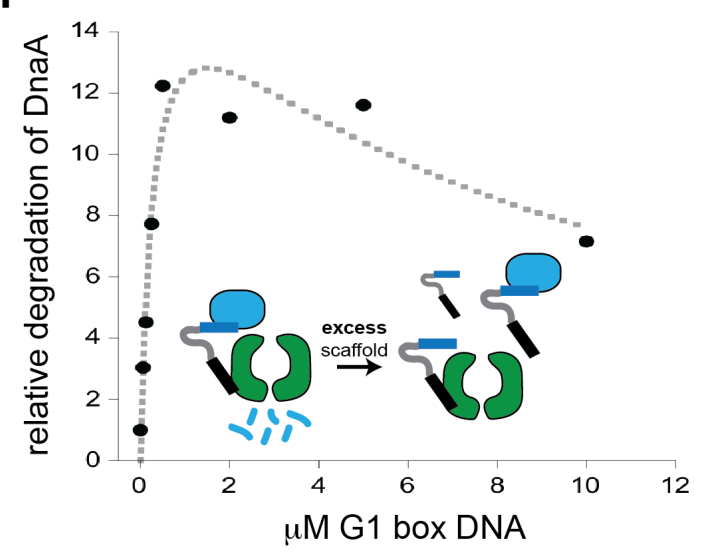


Figure 1. DNA binding affects Lon activity directly and as an adaptor. A. Genomic DNA (gDNA), single strand oligonucleotides (ssDNA) or double-stranded annealed oligonucleotides or PCR products (dsDNA) have different effects on Lon degradation of the model substrate casein. B. Different oligonucleotide sequences can either activate or inhibit Lon protease activity. C. Inhibitory activity of oligonucleotide OPC498 which contains a $\mathrm{G}$ tetrad $(\mathrm{G} 4)$ prone region is dependent on the presence of the $\mathrm{G} 4$ region, but not other secondary structures. D. Schematic to show DnaA binds specifically to $G$ box containing DNA and dsDNA constructs used to test adaptor activity. E. dsDNA capable of binding both DnaA and Lon facilitates rapid degradation of DnaA, which is normally slowly degraded in vitro in these conditions. F. DnaA degradation was monitored as a function of increasing dsDNA G1 box adaptor. Inset shows prediction of reduced ternary complex formation due to excess scaffold and partial site binding. 
Figure 2

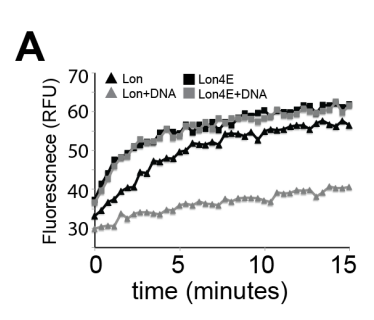

B
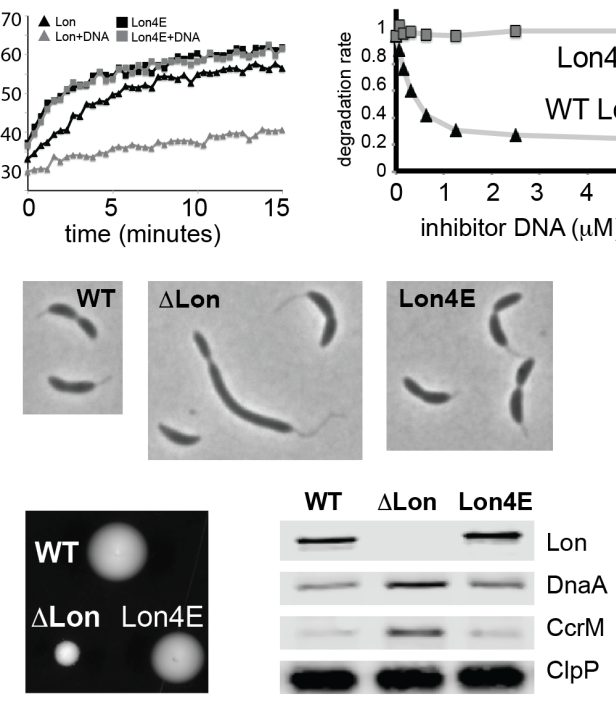

C
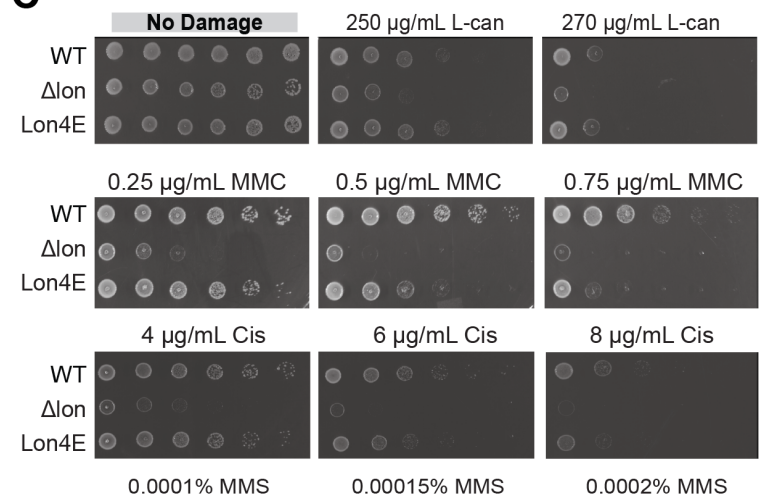

$6 \mu \mathrm{g} / \mathrm{mL}$ Cis

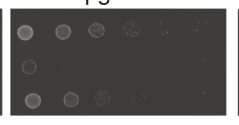

$0.00015 \%$ MMS

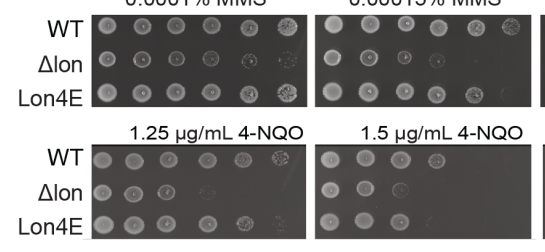

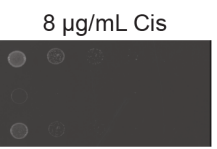

$0.0002 \% \mathrm{MMS}$

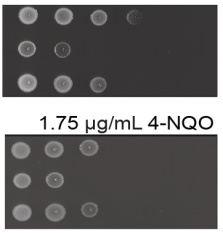

Figure 2. DNA binding mutants of Caulobacter Lon rescue normal growth but not DNA damage tolerance. A. Lon4E degrades FITC-Casein as well as WT Lon, but fails to respond to DNA. B. Morphology, stalk length, and motility defects of cells lacking Lon are not seen in Lon4E strains (see Figure S2 for additional details). Levels of known Lon substrates (CcrM and DnaA) are elevated in $\Delta$ lon cells $(\mathrm{CcrM}=245 \%+/-9 \%$; DnaA $=$ $162 \%+/-5 \%$ of WT; $n=3)$, but mostly reduced to WT levels in the Lon4E mutant (CcrM = $128+/-10 \%$ of WT, DnaA $=116+/-2 \%$ of WT; $n=3$ ). C. Lon4E results in sensitivity to genotoxic stresses. Serial dilutions (10-fold dilutions left to right) of wildtype (WT), Lon deficient $(\Delta / o n)$, or DNA binding deficient Lon expressing (Lon4E) strains on media containing various concentrations of either proteotoxic (L-canavanine (L-can)) or genotoxic (mitomycin C (MMC), cisplatin (Cis), methyl methanosulfonate (MMS), 4nitroquinolone (4NQO)) compounds. 
Figure 3

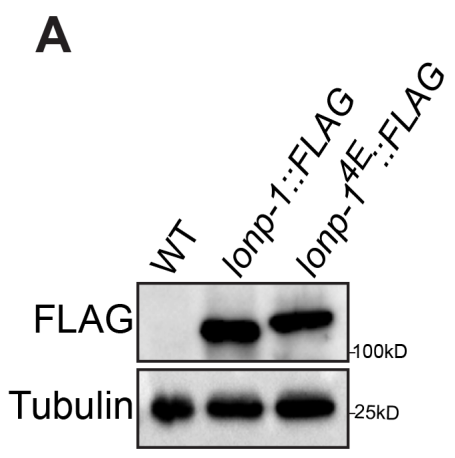

B amount of DNA from $\alpha$-FLAG IP (normalized to WT)

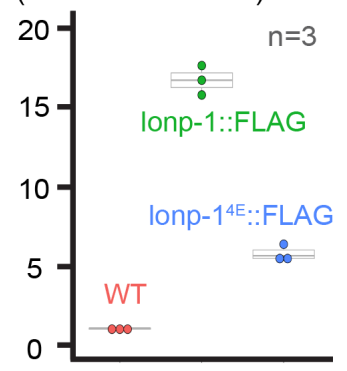

C

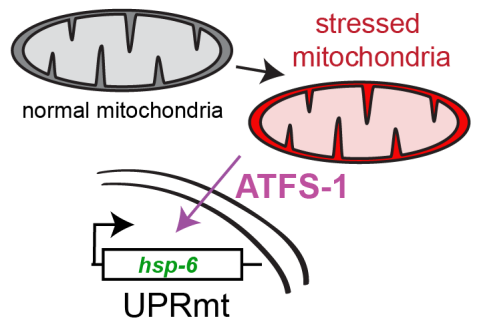

\section{D $0 \mu \mathrm{g} / \mathrm{ml}$ Cis}
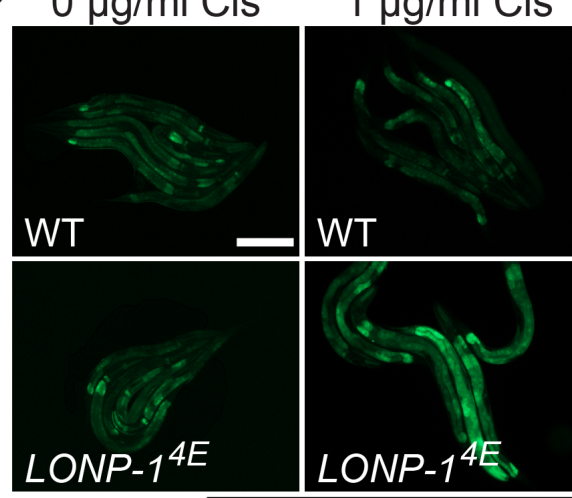

$10 \mu \mathrm{g} / \mathrm{ml}$ Cis

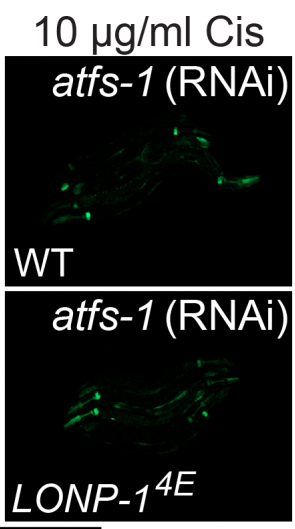

$h s p-6_{p r}: g f p$




Figure 3. DNA binding mutants of mitochondria Lon restore normal development but are sensitive to DNA damaging conditions. A. Immunoblots of lysates from WT worms, or strains expressing LONP-1-FLAG or LONP- $1^{4 E}-F L A G$. Tubulin serves as a loading control. B. ChIP of mtDNA with FLAG antibody in WT, LONP-1-FLAG and LONP-1 ${ }^{4 E}$ FLAG strains. C. Cartoon illustrating hsp-6 reporter of mitochondria stress. D.

Photomicrographs of $h s p-6_{p r::}: g f p$ WT or LONP $-1^{4 \mathrm{E}}$ worms raised on DMSO or cisplatin, scale bar, $0.5 \mathrm{~mm}$. E. Photomicrographs of $h s p-6_{p r}: . g f p$ WT or LONP- $1^{4 E}$ worms raised on paraquat (PQ) or spg-7(RNAi). 


\section{Figure 4}

A

+ inducer

$\Delta$ lon Pxyl::Ion

$\triangle \mathrm{uvrD}$

$\Delta$ lon Pxyl::Ion

$\Delta$ uvrD

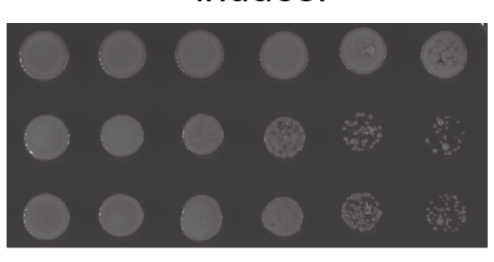

no inducer

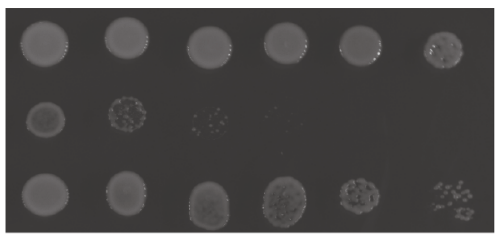

B

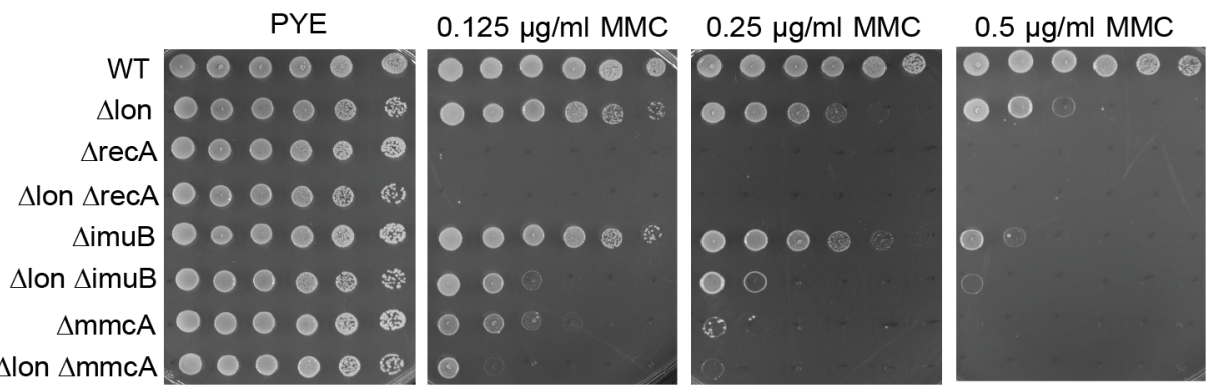

C

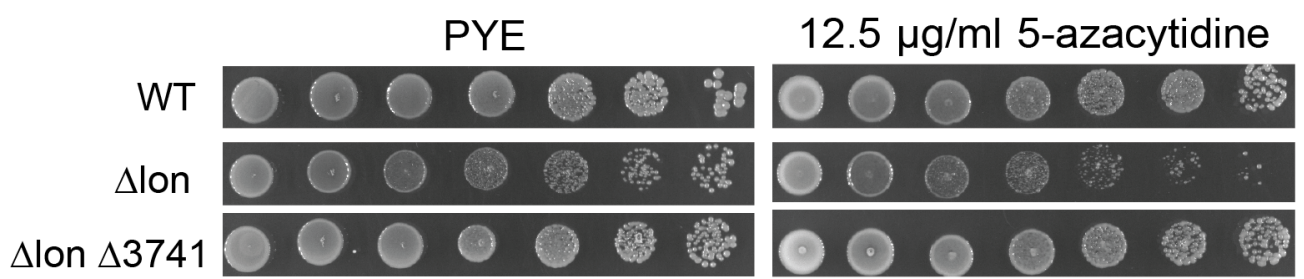

D

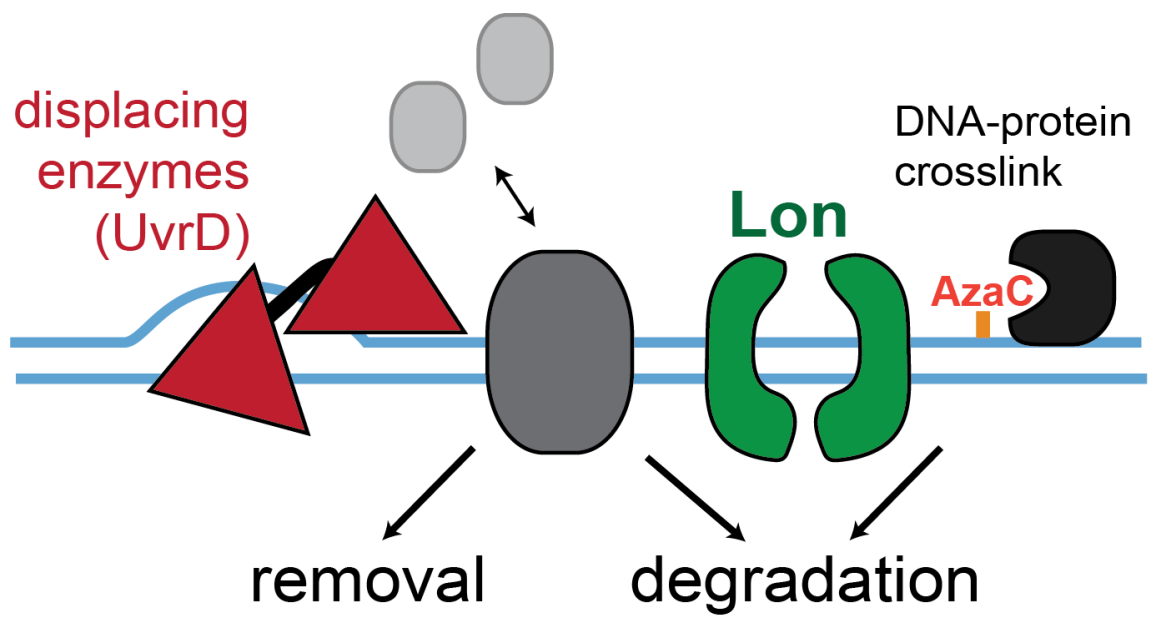


Figure 4. DNA binding of Lon promotes DNA-bound protein clearance. A. Depletion of Lon in $\Delta u v r D$ strains carrying a xylose inducible Lon (Pxyl::Lon) as the sole copy in the cell results in reduced fitness. B. Ion shows no epistasis with other components of DNA damage response such as $\operatorname{rec} A, i m u B$, and $m m c A$. C. $\Delta / o n$ strains are sensitive to $5-$ azacytidine, which generates toxic DNA-protein adducts due to crosslinking of cytosine methyltransferases. Deletion of the cytosine methyltransferase CCNA_03741 $(\Delta 3741)$ in a $\Delta / o n$ background reduces toxicity. D. DNA binding proteins dwell on DNA for a specific amount of time, but if they are overly persistent, must be removed by either displacing enzymes, such as UvrD, which remove them through eviction, or Lon can also degrade these complexes. This role is particularly important for degrading covalent DNA-protein crosslinks, such as those generated by 5-azacytidine (AzaC). 


\section{Acknowledgements:}

We thank Lucy Shapiro for supplying the anti-CcrM antibody and members of the Chien lab and the Protein Homeostasis theme of the Center for Models to Medicine for helpful feedback. Funding: This work was supported by HHMI (C.M.H.), the Mallinckrodt Foundation (C.M.H) and National Institutes of Health grants (R01AG040061) to C.M.H. and (R01GM111706) to P.C. R.Z. and J.L. were funded in part by the UMass Chemistry Biology Interface Training Program (T32GM0515). Author contributions: P.C., J.L., and R.Z. conceptualized the study. J.L., R.Z, Q.Y., and Y.D. performed experiments. P.C., C.H., R.Z, and J.L wrote the initial draft. All authors reviewed the final draft. Data and materials availability: All data is available in the main text or the supplementary materials.

\section{Supplementary Materials:}

Materials and Methods

Figures S1-S3

Tables S1-S2 


\section{References and Notes}

1. P. D. Curtis, Y. V. Brun, Getting in the loop: regulation of development in Caulobacter crescentus. Microbiol Mol Biol Rev 74, 13-41 (2010).

2. K. K. Joshi, P. Chien, Regulated Proteolysis in Bacteria: Caulobacter. Annu Rev Genet 50, 423-445 (2016).

3. U. Jenal, T. Fuchs, An essential protease involved in bacterial cell-cycle control. The EMBO journal 17, 5658-5669 (1998).

4. K. K. Joshi, M. Berge, S. K. Radhakrishnan, P. H. Viollier, P. Chien, An Adaptor Hierarchy Regulates Proteolysis during a Bacterial Cell Cycle. Cell 163, 419-431 (2015).

5. J. Lau, L. Hernandez-Alicea, R. H. Vass, P. Chien, A Phosphosignaling Adaptor Primes the AAA+ Protease CIpXP to Drive Cell Cycle-Regulated Proteolysis. Mol Cell 59, 104-116 (2015).

6. P. T. McGrath, A. A. Iniesta, K. R. Ryan, L. Shapiro, H. H. McAdams, A dynamically localized protease complex and a polar specificity factor control a cell cycle master regulator. Cell 124, 535-547 (2006).

7. S. C. Smith et al., Cell cycle-dependent adaptor complex for ClpXP-mediated proteolysis directly integrates phosphorylation and second messenger signals. Proceedings of the National Academy of Sciences of the United States of America 111, 14229-14234 (2014).

8. A. A. Iniesta, P. T. McGrath, A. Reisenauer, H. H. McAdams, L. Shapiro, A phospho-signaling pathway controls the localization and activity of a protease complex critical for bacterial cell cycle progression. Proceedings of the National Academy of Sciences of the United States of America 103, 10935-10940 (2006).

9. A. Duerig et al., Second messenger-mediated spatiotemporal control of protein degradation regulates bacterial cell cycle progression. Genes \& development 23 , 93-104 (2009).

10. R. Wright, C. Stephens, G. Zweiger, L. Shapiro, M. R. Alley, Caulobacter Lon protease has a critical role in cell-cycle control of DNA methylation. Genes \& development 10, 1532-1542 (1996).

11. K. G. Gora et al., Regulated proteolysis of a transcription factor complex is critical to cell cycle progression in Caulobacter crescentus. Molecular microbiology 87 , 1277-1289 (2013).

12. K. Jonas, J. Liu, P. Chien, M. T. Laub, Proteotoxic stress induces a cell-cycle arrest by stimulating Lon to degrade the replication initiator DnaA. Cell 154, 623636 (2013).

13. A. L. Goldberg, Degradation of abnormal proteins in Escherichia coli (protein breakdown-protein structure-mistranslation-amino acid analogs-puromycin). Proceedings of the National Academy of Sciences of the United States of America 69, 422-426 (1972).

14. E. Gur, R. T. Sauer, Recognition of misfolded proteins by Lon, a AAA(+) protease. Genes \& development 22, 2267-2277 (2008).

15. E. M. Witkin, Inherited Differences in Sensitivity to Radiation in Escherichia Coli. Proceedings of the National Academy of Sciences of the United States of America 32, 59-68 (1946). 
16. B. F. Johnson, J. Greenberg, Mapping of sul, the suppressor of lon in Escherichia coli. J Bacteriol 122, 570-574 (1975).

17. S. Mizusawa, S. Gottesman, Protein degradation in Escherichia coli: the lon gene controls the stability of sulA protein. Proceedings of the National Academy of Sciences of the United States of America 80, 358-362 (1983).

18. D. A. Bota, K. J. Davies, Lon protease preferentially degrades oxidized mitochondrial aconitase by an ATP-stimulated mechanism. Nat Cell Biol 4, 674680 (2002).

19. A. M. Nargund, M. W. Pellegrino, C. J. Fiorese, B. M. Baker, C. M. Haynes, Mitochondrial import efficiency of ATFS-1 regulates mitochondrial UPR activation. Science 337, 587-590 (2012).

20. B. Lu et al., Roles for the human ATP-dependent Lon protease in mitochondrial DNA maintenance. J Biol Chem 282, 17363-17374 (2007).

21. B. A. Zehnbauer, E. C. Foley, G. W. Henderson, A. Markovitz, Identification and purification of the Lon+ (capR+) gene product, a DNA-binding protein.

Proceedings of the National Academy of Sciences of the United States of America 78, 2043-2047 (1981).

22. C. H. Chung, A. L. Goldberg, The product of the lon (capR) gene in Escherichia coli is the ATP-dependent protease, protease La. Proceedings of the National Academy of Sciences of the United States of America 78, 4931-4935 (1981).

23. M. F. Charette, G. W. Henderson, L. L. Doane, A. Markovitz, DNA-stimulated ATPase activity on the lon (CapR) protein. J Bacteriol 158, 195-201 (1984).

24. C. H. Chung, A. L. Goldberg, DNA stimulates ATP-dependent proteolysis and protein-dependent ATPase activity of protease La from Escherichia coli. Proceedings of the National Academy of Sciences of the United States of America 79, 795-799 (1982).

25. T. Liu et al., DNA and RNA binding by the mitochondrial lon protease is regulated by nucleotide and protein substrate. J Biol Chem 279, 13902-13910 (2004).

26. N. Maizels, L. T. Gray, The G4 genome. PLoS Genet 9, e1003468 (2013).

27. S. H. Chen, C. K. Suzuki, S. H. Wu, Thermodynamic characterization of specific interactions between the human Lon protease and G-quartet DNA. Nucleic Acids Res 36, 1273-1287 (2008).

28. L. Ambro, V. Pevala, J. Bauer, E. Kutejova, The influence of ATP-dependent proteases on a variety of nucleoid-associated processes. J Struct Biol 179, 181192 (2012).

29. G. K. Fu, M. J. Smith, D. M. Markovitz, Bacterial protease Lon is a site-specific DNA-binding protein. J Biol Chem 272, 534-538 (1997).

30. I. Lee, C. K. Suzuki, Functional mechanics of the ATP-dependent Lon proteaselessons from endogenous protein and synthetic peptide substrates. Biochim Biophys Acta 1784, 727-735 (2008).

31. J. A. Taylor, M. C. Ouimet, R. Wargachuk, G. T. Marczynski, The Caulobacter crescentus chromosome replication origin evolved two classes of weak DnaA binding sites. Molecular microbiology 82, 312-326 (2011).

32. A. Karlowicz et al., Defining the crucial domain and amino acid residues in bacterial Lon protease for DNA binding and processing of DNA-interacting substrates. J Biol Chem 292, 7507-7518 (2017).

33. N. Chatterjee, G. C. Walker, Mechanisms of DNA damage, repair, and mutagenesis. Environ Mol Mutagen 58, 235-263 (2017).

34. L. Margulis, Origin of eukaryotic cells; evidence and research implications for a theory of the origin and evolution of microbial, plant, and animal cells on the Precambrian earth. (Yale University Press, New Haven,, 1970), pp. xxii, 349 p. 
35. M. W. Gray, Mitochondrial evolution. Cold Spring Harb Perspect Biol 4, a011403 (2012).

36. T. Yoneda et al., Compartment-specific perturbation of protein handling activates genes encoding mitochondrial chaperones. J Cell Sci 117, 4055-4066 (2004).

37. H. Ogawa, K. Shimada, J. Tomizawa, Studies on radiation-sensitive mutants of

E. coli. I. Mutants defective in the repair synthesis. Mol Gen Genet 101, 227-244 (1968).

38. V. Petrova et al., Active displacement of RecA filaments by UvrD translocase activity. Nucleic Acids Res 43, 4133-4149 (2015).

39. X. Veaute et al., UvrD helicase, unlike Rep helicase, dismantles RecA nucleoprotein filaments in Escherichia coli. The EMBO journal 24, 180-189 (2005).

40. V. Epshtein et al., UvrD facilitates DNA repair by pulling RNA polymerase backwards. Nature 505, 372-377 (2014).

41. D. V. Santi, A. Norment, C. E. Garrett, Covalent bond formation between a DNAcytosine methyltransferase and DNA containing 5-azacytosine. Proceedings of the National Academy of Sciences of the United States of America 81, 69936997 (1984).

42. J. B. Kozdon et al., Global methylation state at base-pair resolution of the Caulobacter genome throughout the cell cycle. Proceedings of the National Academy of Sciences of the United States of America 110, E4658-4667 (2013).

43. J. Stingele, B. Habermann, S. Jentsch, DNA-protein crosslink repair: proteases as DNA repair enzymes. Trends Biochem Sci 40, 67-71 (2015).

44. J. M. Skerker, M. S. Prasol, B. S. Perchuk, E. G. Biondi, M. T. Laub, Twocomponent signal transduction pathways regulating growth and cell cycle progression in a bacterium: a system-level analysis. PLoS Biol 3, e334 (2005).

45. B. Ely, R. C. Johnson, Generalized Transduction in CAULOBACTER CRESCENTUS. Genetics 87, 391-399 (1977).

46. E. F. Vieux, M. L. Wohlever, J. Z. Chen, R. T. Sauer, T. A. Baker, Distinct quaternary structures of the AAA+ Lon protease control substrate degradation. Proceedings of the National Academy of Sciences of the United States of America 110, E2002-2008 (2013).

47. C. M. Haynes, Y. Yang, S. P. Blais, T. A. Neubert, D. Ron, The matrix peptide exporter HAF-1 signals a mitochondrial UPR by activating the transcription factor ZC376.7 in C. elegans. Mol Cell 37, 529-540 (2010).

48. A. Paix, A. Folkmann, G. Seydoux, Precision genome editing using CRISPRCas9 and linear repair templates in C. elegans. Methods 121-122, 86-93 (2017).

49. I. Katic, L. Xu, R. Ciosk, CRISPR/Cas9 Genome Editing in Caenorhabditis elegans: Evaluation of Templates for Homology-Mediated Repair and Knock-Ins by Homology-Independent DNA Repair. G3 (Bethesda) 5, 1649-1656 (2015).

50. A. E. Friedland et al., Heritable genome editing in C. elegans via a CRISPR-Cas9 system. Nat Methods 10, 741-743 (2013).

51. A. M. Nargund, C. J. Fiorese, M. W. Pellegrino, P. Deng, C. M. Haynes, Mitochondrial and nuclear accumulation of the transcription factor ATFS-1 promotes OXPHOS recovery during the UPR(mt). Mol Cell 58, 123-133 (2015).

52. A. Ducret, E. M. Quardokus, Y. V. Brun, MicrobeJ, a tool for high throughput bacterial cell detection and quantitative analysis. Nat Microbiol 1, 16077 (2016).

53. H. Li, R. Durbin, Fast and accurate long-read alignment with Burrows-Wheeler transform. Bioinformatics 26, 589-595 (2010).

54. H. Li et al., The Sequence Alignment/Map format and SAMtools. Bioinformatics 25, 2078-2079 (2009). 
55. A. R. Quinlan, BEDTools: The Swiss-Army Tool for Genome Feature Analysis. Curr Protoc Bioinformatics 47, 1112 11-34 (2014).

56. M. D. Robinson, D. J. McCarthy, G. K. Smyth, edgeR: a Bioconductor package for differential expression analysis of digital gene expression data. Bioinformatics 26, 139-140 (2010). 